\title{
BOUND STATES OF VECTOR BOSONS
}

\author{
M. VELTMAN \\ Physics Department, University of Michigan, Ann Arbor, MI 48109, USA
}

Received 2 February 1984

The anomalous CERN events are interpreted as a new state decaying into a photon and a lepton pair. It is speculated that this new state might be a composite state involving vector bosons.

\section{Introduction. Recently the CERN UAl and} UA2 experiments have found evidence for the existence of both the neutral and the charged vector bosons of weak interactions [1]. The observed results are for the most part in excellent agreement with the standard model, in particular the measured values for the masses. Also the $\rho$-parameter has been measured to be very close to one.

Yet the observed decay of the neutral $W$ into leptons + photon is by far too large to fit the predictions of the standard model. Of course, it is too early to decide definitely on this issue: it may well be that the effect disappears when more data becomes available. On the other hand, the observation of these decay modes in both UA1 and UA2 with electrons and muons is really striking.

In this note we accept these events at face value, and will try to analyze the consequences of this. In addition we will include in our considerations another strange fact: the occurrence of same-sign di-leptons and other multi-lepton combinations in neutrino reactions [2]. These events have been known for some time, and nobody has been able to come up with a satisfactory explanation. Again, the data are not very strong at this time, and also this effect may be some kind of statistical or experimental fluke. But again, the events have been observed in different experiments, and it becomes difficult to ignore them.

2. Neutral $W$ decay. The first idea that comes to mind is that the neutral vector boson decays into a photon and a lepton pair with a rather strong coupling, not present in the standard model. This could be interpreted as a manifestation of a non-local structure of the vector bosons, and indeed this possibility has been suggested recently [3]. However, there are a number of objections against this possibility. First of all, if this were the case, then we would also expect a substantial decay of the charged W's into leptons and a photon. This has not been observed, to the level of a few percent [4]. Secondly, a structure for the vector bosons would very likely result in a difference in measured mass of the vector bosons when measured at low energy or when observed at high $(=90 \mathrm{GeV})$ energy. The perfect agreement of the observed mass with predictions from the low-energy data show that there is virtually no momentum dependence in the vector-boson self-energy, which effectively means that the vector bosons cannot have strong coupling. They must remain point-like to at least $90 \mathrm{GeV}$. Finally, if the neutral vector boson is differently coupled as compared to the charged $W$ then it is very hard to understand why the $p$-parameter comes out so close to one. In short, tampering with the standard model on this level is almost impossible. Also it is very difficult to write any kind of model for this situation, where perturbation theory holds very well at low energies. For instance, what to do with graphs as shown in fig. 1 , whose effects could be very large, as the $W$-lepton coupling is presumably as strong as the W-lepton-photon coupling. It should be noted, however, that this problem is there in all cases, even if the CERN events have nothing to do with vector bosons. 

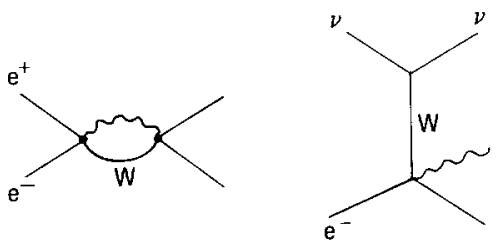

Fig. 1.

As an example of a theory with strongly interacting bosons we may introduce a new neutral scalar particle, interacting strongly with the vector bosons. This particle could then be used, one way or the other, to be responsible for the non-standard decay modes. By taking this particle to be neutral, and coupling it with the correct relative strength to the neutral and charged vector bosons we may avoid trouble with the $\rho$ parameter. However, this seems to lead to a violation of gauge invariance, and moreover we still do get into trouble with the observed masses, agreeing with the perturbative calculations using as input low-energy data. To show this we will introduce a model, showing a construction that is generally useful here. The aim is to introduce non-standard couplings, while leaving gauge invariance unchanged. Also the couplings should have as little effect as possible on the renormalization procedure. We will require renormalizability to at least one loop.

Thus we consider the usual standard model, described by a lagrangian made up from several parts:

$$
\begin{aligned}
L & =L(\mathrm{~W})+L(\text { ferm })+L \text { (Higgs })+L \text { (Higgs, fermions }) \\
& +L(\text { g.b. })+L \text { (F.P. })
\end{aligned}
$$

We will not consider quantum chromodynamics, which might be a mistake. Now introduce a scalar neutral particle $S$, and modify the lagrangian by adding a part quadratic in $\mathrm{S}$, with mass $M_{\mathrm{S}}$, and replacing $L$ (Higgs) by $(1+h \cdot \mathrm{S}) \cdot L$ (Higgs). In this $h$ is some coupling constant, presumably large. This leaves gauge invariance untouched, but there are now some non-renormalizable couplings, for instance WWHHS. If we assume the Higgs particle to be quite heavy $(>100 \mathrm{GeV})$ then practically none of the new couplings are of any consequence, with the exception of the WWS couplings. Also we can do our calculations as usual in some "renormalizable" gauge.

However, this model is not acceptable. The lagrangian contains the interactions

$$
h M^{2} \mathrm{SW}^{+} \mathrm{W}^{-}+h\left(M^{2} / c^{2}\right) \mathrm{SW}^{0} \mathrm{~W}^{0} \text {. }
$$

$\mathrm{W}^{+}$and $\mathrm{W}^{0}$ self-energy graphs, in particular the infinite parts, are in the ratio $c^{4}$, thus leading to (infinite) corrections to the $\rho$-parameter.

On the basis of the above arguments we will now abandon the idea that we are dealing with a new direct or indirect relatively strong coupling of leptons and photons with the vector bosons.

3. Phenomenology. On the pure phenomenological level the following may be noted. If we insist that the electromagnetic interactions are basically minimal (i.e. derive from the combination $\mathrm{D}_{\mu}=\partial_{\mu}+\mathrm{i} e A$ ), then the occurrence of a process involving a photon must necessarily imply the occurrence of the same process without a photon but about a factor $\alpha$ stronger. The only way to avoid this is to arrange the coupling such that the process without a photon is forbidden. This may be achieved by introducing terms involving commutators of derivatives; $\left[D_{\mu}, D_{\nu}\right]$ is zero if $D_{\mu}=\partial_{\mu}$. In fact, the well-known Pauli magnetic moment can be written in this way, and similar possibilities exist with vector bosons. If we do not accept an exceedingly large magnetic moment for the vector bosons themselves (which we indeed do not), then we must conclude that the photon couples to some magnetic moment of dynamic origin, much like for instance the $\pi^{0} \rightarrow 2 \gamma$ coupling. Such couplings involve $F_{\mu \nu}$ rather than $A_{\mu}$ directly. From here on we do not have much choice: it seems fairly impossible to view the CERN observed results as some lowest order process.

We thus take the point of view that the CERN events are the manifestation of a new object, probably compound, with as yet undetermined properties. Not much can be said, except that none of the presently popular theories seems particularly adapted to this situation.

4. Absence of Higgs. As we have pointed out repeatedly there are grave doubts about the Higgs system [5], and we would like to take up again the line of speculation that there is no such thing as a Higgs. As noted before this implies that vector bosons with energies over $1 \mathrm{TeV}$ would have strong interactions, but there are practically no consequences at low 
energy. We refer here to the screening theorem [6].

However the situation may change dramatically if these new strong interactions produce low lying bound states [6] or quasi-confined states. It is tempting to interpret the CERN events in this way: there is a bound state of two vector bosons with a mass of approximately $90 \mathrm{GeV}$. It would be natural to assign to this particle the properties of the channel in which W-interactions become strong above $1 \mathrm{TeV}$ : S-wave scattering of longitudinally polarized W's with isospin zero, but we have really no sound arguments here. In fact, the complete absence of any theory of such strong interactions forbids more than qualitative arguments. It should be noted that several groups have tried to develop this kind of strong interaction theory, but without substantial success [7].

How would an object of the type mentioned manifest itself? It would mainly behave as a combination of two vector bosons (both $\mathrm{W}^{+} \mathrm{W}^{-}$and $\mathrm{W}^{0} \mathrm{~W}^{0}$ combinations since the $I$-spin is 0 ), and consequently act as an effective eight-fermion type interaction. Notable consequences of this model are:

- UA1 and UA2 should also observe events of the type $\mathrm{e}^{+} \mathrm{e}^{-} \nu \bar{\nu}$ or $\gamma \nu \bar{\nu}^{\neq 1}$;

- there should also be events of the type hadrons +hard photon;

- there might be two-photon decay modes ${ }^{\neq 1}$.

In addition, there might be observable consequences in high energy neutrino interactions. The basic process might be due to diagrams as sketched in fig. 2 . The final state could contain as many as 5 charged leptons, but these would be rare as the lepton/hadron decay ratio of vector bosons is about $1 / 3$. Also hard photon decays might be significant. Such things could perhaps be seen also at PEP and PETRA. As a matter of fact

${ }^{\ddagger 1}$ This depends on the particular $\mathrm{SU}_{2} \times \mathrm{U}_{1}$ structure of the composite state.
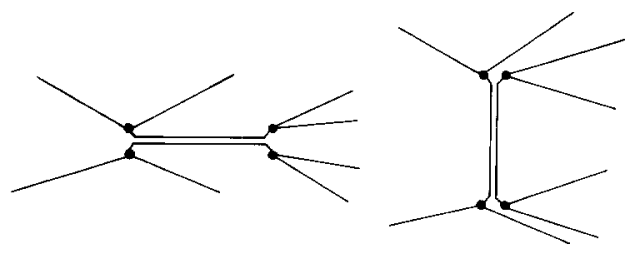

Fig. 2 . events of this type have been observed at a rate exceeding that predicted by any other known mechanism.

The qualitative predictions of this model agree reasonable well with the observed data in neutrino interactions. As it happens many channels are obscured by compeing conventional processes involving charm production etc., but a few escape this fate, notably the same-sign di-leptons and tri-leptons. There are even some tetra-lepton events, very hard to explain by any conventional mechanism.

The author is deeply indebted to Dr. R. Thun and Dr. S. Errede, for stimulating discussions on the exotic neutrino and $\mathrm{e}^{+} \mathrm{e}^{-}$interactions. Also informative discussions with Dr. Yovanovich and Dr. F. Sculli and helpful criticism and comments of Dr. M. Duncan, Dr. G. Kane, and Dr. R. Philippe are gratefully acknowledged.

\section{References}

[1] UA1 Collab., G. Arnison et al., Phys. Lett. 122B (1983) 103 ;

UA2 Collab., M. Banner et al., Phys. Lett. 122B (1983)

476;

UAl Collab., G. Arnison et al., Phys. Lett. 126B (1983)

398 ;

UA2 Collab., P. Bagnaia et al., Phys. Lett. 129B (1983)

130 ;

UA1 Collab., G. Arnison et al., Phys. Lett. 129B (1983) 273.

[2] T. Nash, Intern. Symp. on Lepton and photon interactions (Cornell, August 1983);

H.E. Fisk, Intern. Symp. on Lepton and photon interactions (Bonn, August 1981).

[3] G. Gounaris, R. Kogerler and D. Schildknecht, University of Bielefeld preprint (October 1983).

[4] UA1 Collab., G. Arnison et al., Phys. Lett. 135B (1984) 250.

[5] M. Veltman, Proc. Intern. High energy Conf. (Brighton, July 1983).

[6] M. Veltman, Acta Physica Polonica B8 (1977) 475; Phys. Lett. 70B (1977) 254;

T. Appelquist and R. Shankar, Nucl. Phys. B158 (1979) 317;

T. Appelquist and C. Bernhard, Phys. Rev. D22 (1980) 200 ;

A.C. Longhitano, Phys. Rev. D22 (1980) 1166.

[7] B.W. Lee, C. Quigg and H. Thacker, Phys. Rev. Lett. 38 (1977) 883 